\title{
Evacuation Simulation Of Passenger Ship Based On Cellular Automata
}

\author{
Min Hu, Wei Cai \\ School of Transportation, Wuhan University of Technology, Wuhan 430063, China.
}

Keywords: the passenger ship; cellular automata; static floor field; dynamic floor field; evacuation efficiency

\begin{abstract}
In this paper, the cellular automata model is applied to the passenger evacuation in the passage ship. The distance between the cell and the exit is calculated by coordinate difference method, and the impact factors of the static floor field on passengers are obtained. At the same time, the attraction of the mainstream crowd and the repulsion impact among passengers are taken into consideration, and then the impact factors of the dynamic floor field are obtained. The transition probability of passenger at any place is calculated by weighting influencing factors of the static floor field and the dynamic floor field. Then attraction factors for the global cell is established. The evacuation process is simulated with $\mathrm{VC}+$ + and the total of evacuation time steps is obtained. Moreover, the influence of factors on the evacuation is discussed.
\end{abstract}

\section{Introduction}

Nearly a decade of global data show that there are many causes of marine accidents, such as collision, fire, explosion, stranded, bad weather, and so on. The mortality rate of passenger ships is higher than other ships. The passenger ship evacuation problem has become the focus of the world's shipping industry.

Until now, extensive research on micro and macro evacuation models has facilitated the development of emergency evacuation areas, and the impact of passengers' physiological and psychological factors on evacuation has been proposed. The social force model proposed by Helbing [1], the lattice gas model put forward by Rothman and Zaleski [2] and the cellular automata model suggested and developed respectively by Blue and Adler [3-4] as well as Muramatsu and Nagatani [5] et al. In terms of evacuation, Considering the influence of aisles in the classroom, Zhu Kongjin et al. [6] established cellular automata evacuation model to analyze the evacuation efficiency with different exits and layouts of the room. Yue Hao [7] put forward a cellular automata model describing the passengers walking based on four dynamic parameters to simulate the oppositedirectional flow of passengers.

In terms of passenger evacuation in ships, $\mathrm{Ha}$ [8] et al. established an evacuation model based on the cellular automata theory while considering the impact of the crowd movement in opposite directions on the evacuation time. Vanem [9] et al. simulated the evacuation from a ro-ro passenger ship based on the IMO criterion. Considering the impact of rolling on the escape speed, Kim [10] et al. carried out a simulation based on the IMEX (Intelligent Model for Extrication Simulation) system. Yoshida et al. [11] studied the evacuation speed of individuals with different genders and heights when rolling and pitching are involved.

This paper has three additional sections. The next section describes the detailed process of evacuation model establishment. Section 3 presents simulation of the evacuation process. Finally, section 4 concludes the work.

\section{Establishment of an evacuation model from the passenger ship}

The cellular automata has been widely used to simulate natural phenomena such as motion, diffusion, spread and evolution. It also has been extensively applied to traffic flow and crowd evacuation simulation. As a scalable multidimensional system with space and time both discrete, its simulation space consists of a series of cells and each cell has a limited number of states, with the local rules adopted to update all cellular states in the system. The cellular automata model doesn't conform to a unified physical model or mathematical equation, and the states and interaction of the whole system are realized based on local rules. Therefore, the cellular automata feature such advantages as algorithm simplicity, high computational efficiency and easy understanding.

\subsection{Mesh generation of the evacuation space}

The basic framework of the model is to divide the plane into even cells with square being the most common shape. Each cell is taken up by walls, other obstacles, passengers, or kept empty. The crowd move among the cells in order to realize the motion process and each cell can be occupied by one passenger at most.

The mesh generation shows the static component of the system while the cellular automata is a dynamic one which is discrete in time. Namely, the time is changing isochronally and the states of all cells are simultaneously changing at every moment. The time of each synchronous change is called a time step. 
In the cellular automata, these rules are defined in a local space, namely, the state of a cell in the next moment is decided by the states of the cell itself and its neighboring cells. Therefore, before specified by the rules, it is necessary to clearly define the neighboring rules and to clarify which cells are neighbors of this cell. Figure 1 shows several common neighborhood types, with the yellow and green cells as the central and neighboring ones respectively. Under the Von Neumann neighborhood, each passenger at each time step can have four optional positions as the targets for the next step, that is to say, the passenger can wait at in-situ or move towards one of the four positions around. In the Moore neighborhood or the extended Moore neighborhood, each passenger has eight optional positions. In the extended Moore neighborhood, the number of neighbors for cell is $\left((2 r+1)^{d}-1\right)$, where $d$ is the number of dimensions and $r$ is the radius of the neighborhood.

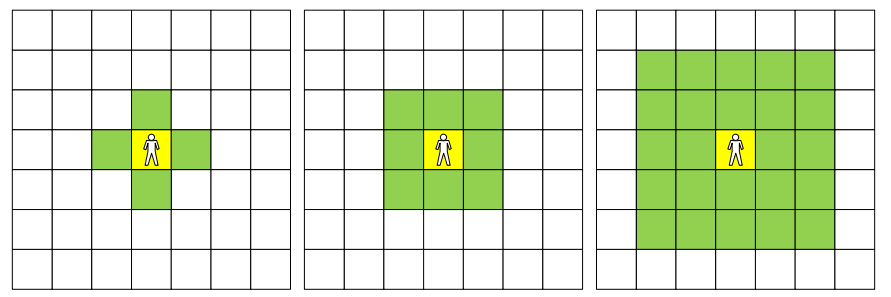

Figure 1: Types of neighborhoods.

The typical size of a cell in the cellular automata is $40 \mathrm{~cm} \mathrm{x} 40$ $\mathrm{cm}$. If all cells are occupied by passengers, the density would be 6.2 persons $/ \mathrm{m}^{2}$. Another common size is $50 \mathrm{~cm} \times 50 \mathrm{~cm}$ with maximum density of 4 persons $/ \mathrm{m}^{2}$. Studies [12] have shown that when density reaches $4-5.5$ persons $/ \mathrm{m}^{2}$, the crowd is just able to move. If density increases, the crowd will hardly be able to move. Considering the swing of limbs and unwillingness to make physical contact with others, passengers would expect more space than that is occupied by their bodies. In this paper, the $50 \mathrm{~cm} \times 50 \mathrm{~cm}$ cell is adopted.

\subsection{Evacuation model}

The evacuation model includes such two parts as the Static Floor Field and the Dynamic Floor Field. The former is related to the plane space layout of the evacuation while the latter mainly involve the mutual influence among people.

\subsubsection{Static floor field}

The static floor field includes the layout of the evacuation space, and the field intensity is kept constant in the whole process of the evacuation as determined at the beginning. Under normal circumstances, the nearer the cell from the exit, the greater the static floor field intensity will be. When calculating the direction parameters, the distance from the cell location to the emergency exit shall be first calculated and the distance within the emergency exit should be set as zero. Moreover, the coordinate difference method can be used to calculate the relative distance from the cell to the exit.

$$
\mathrm{L}_{i}=\left|C_{i}^{x}-e^{x}\right|+\left|C_{i}^{y}-e^{y}\right|
$$

where $C_{i}$ is the cell whose coordinate is $\left(C_{i}^{x}, C_{i}^{y}\right)$ and $e$ is the exit whose coordinate is $\left(\mathrm{e}^{\mathrm{x}}, \mathrm{e}^{\mathrm{y}}\right) . \mathrm{L}_{\mathrm{i}}$ represents the distance from the cell to the exit. For multi-level emergency exits, the distance of each level shall be superposed for calculation and the above-mentioned formula can be rewritten as:

$$
\mathrm{L}_{i}=\left|C_{i}^{x}-e_{n}^{x}\right|+\left|C_{i}^{y}-e_{n}^{y}\right|+\sum_{j=1}^{n-1}\left(\left|e_{j}^{x}-e_{j+1}^{x}\right|+\left|e_{j}^{y}-e_{j+1}^{y}\right|\right)
$$

where $\mathrm{n}$ indicates the nth layer.

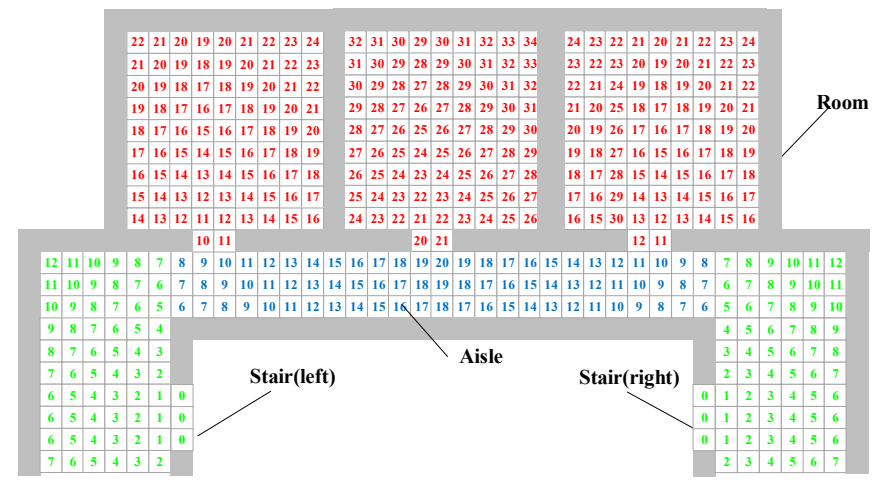

Figure 2: The distance of the cell from the exit.

For an area with orthogonally connections, there is a vision dead zone before turning, so such area is generally divided into two levels for escape. Figure 2 shows the distance value from the room to the stairs, which can be divided into three parts for calculation, including the passenger cabin area, the aisle area and the staircase area, respectively referred to as the third layer, the second layer and the first layer. The results indicate the closer the cell is to the exit, the stronger the attraction of the cell for passengers is. The attraction of the cell for passengers is called attraction factor, which is calculated by formula (3)

$$
F_{i}^{1}=\frac{L_{\max }-L_{i}}{L_{\max }}
$$

In Formula (3), $F_{i}^{l}$ indicates the attraction factor of cell $C_{\mathrm{i}}$ in the passenger cabin and $\mathrm{L}_{\max }$ is the maximum value of the relative global distance. Since the evacuation zone is composed of several areas, when calculating the overall attraction factors, distances of the passenger cabin, aisle and staircase are added up to obtain attraction factor of the static floor field.

\subsubsection{Dynamic floor field}

The dynamic floor field intensity is determined by the crowd movement route and their distribution in the evacuation space, which changes at each time step, mainly including the attraction effect of the mainstream crowd and the mutual exclusivity of individuals.

(1) Attraction effect of the mainstream crowd

Conformity would drive individuals to join the mainstream crowd. The cells that the mainstream crowd passes have an attraction for the following crowds. Two types of neighborhood are established by simulating human vision. As shown in Figure 3 (a) in which there is a five-level neighborhood model, the green cells within the red dotted lines show the ranges occupied by the crowd in four (front, rear, left 
and right) directions within the field of vision. Figure (b) shows the range occupied by the crowd in the diagonal direction.

The crowd movement within the scope of each area can be simulated and the movement direction of the mainstream crowd can be determined by comparing the changes of the crowd movement between two time steps.

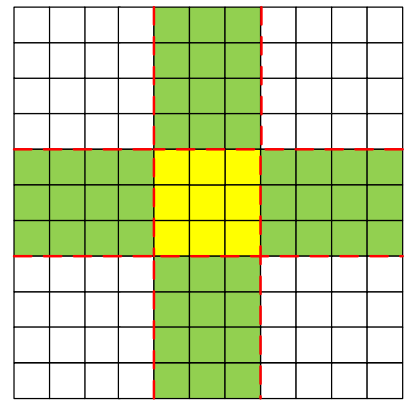

(a)

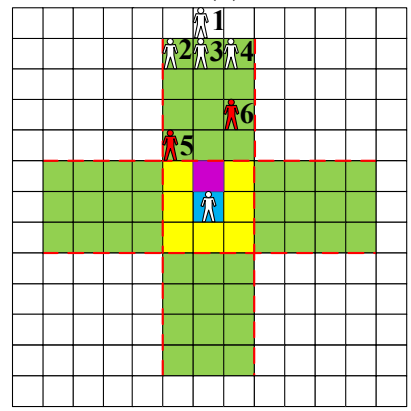

(c)

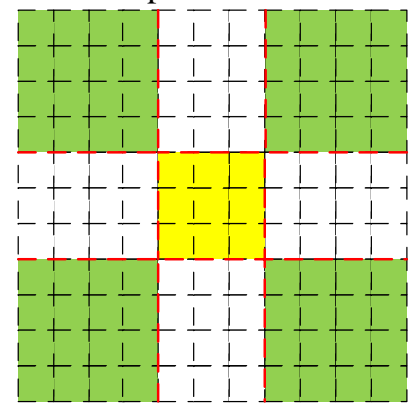

(b)

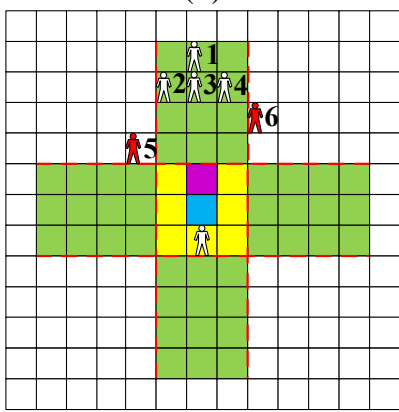

(d)
Figure 3: The area of mainstream crowd.

The blue cells in Figure 3 (c) indicate the positions occupied by the crowd at the current time step and the purple cell shows one of the targets to be evacuated to. Taking the five-level neighborhood model as an example, the attraction factor of the mainstream crowd ahead can be calculated as follows:

$$
\begin{aligned}
& L_{P}^{s u m 1}=\sum_{i=1}^{n}\left(\left|P_{i}^{x 1}-C_{u p}^{x}\right|+\left|P_{i}^{y 1}-C_{u p}^{y}\right|\right) \\
& L_{P}^{\text {sum } 2}=\sum_{i=1}^{n}\left(\left|P_{i}^{x 2}-C_{u p}^{x}\right|+\left|P_{i}^{y 2}-C_{u p}^{y}\right|\right) \\
& F_{u p}^{m}= \begin{cases}1 & L_{P}^{\text {sum } 2}>L_{P}^{\text {sum } 1} \\
0 & L_{P}^{\text {sum } 2}=L_{P}^{\text {sum } 1} \\
-1 & L_{P}^{\text {sum } 2}<L_{P}^{\text {sum } 1}\end{cases}
\end{aligned}
$$

(6)

Firstly, the program refers to the states of all passengers in the previous step .The number $\mathrm{P}_{\mathrm{i}}$ and coordinates of positions $\left(\mathrm{P}_{\mathrm{i}}^{\mathrm{x} 1}\right.$, $\mathrm{P}_{\mathrm{i}}^{\mathrm{y} 1}$ ) of passengers in front of the purple cell are obtained. As shown in Figure 3(d), passengers numbered from 1 to 4 are in front. Then the distances respectively from the four passengers to the front purple cell $\mathrm{C}_{\mathrm{up}}$ with the coordinate $\left(\mathrm{C}_{\mathrm{up}}^{\mathrm{x}}, \mathrm{C}_{\mathrm{up}}^{\mathrm{y}}\right)$ are calculated and finally the sum of the distance values $L_{p}^{\text {sum } 1}$ is obtained.

The program returns to the current moment, according to the serial number $\mathrm{P}_{\mathrm{i}}$ obtained in the first step, the position $\left(\mathrm{P}_{\mathrm{i}}^{\mathrm{x} 2}\right.$, $\mathrm{P}_{\mathrm{i}}^{\mathrm{y}^{2}}$ ) occupied by the passenger at the current moment can be known. Then the distances respectively from the four passengers to the purple cell $\mathrm{C}_{\mathrm{up}}$ with the coordinate $\left(\mathrm{C}_{\mathrm{up}}^{\mathrm{x}}\right.$, $\mathrm{C}_{\mathrm{up}}^{\mathrm{y}}$ ) are calculated, the sum of which is $L_{p}^{s u m 2}$. Meanwhile, the passengers (No.5 and No.6) are not in the front area at the previous moment, so they are not included in the objectives for calculation.

If $F_{u p}^{m}$ is greater than 0 , it indicates that the front crowd is moving far away from the target, which can attract the following passengers, and at this time the attraction factor is 1 for the front cell $\mathrm{C}_{\mathrm{up}}$. If $F_{u p}^{m}$ is smaller than 0 , it means that the front crowd is moving towards the target, which has a repulsive force, and the attraction factor $\mathrm{C}_{\text {up }}$ is -1 . When $F_{u p}^{m}$ is equal to 0 , the crowd does not move and the attraction factor $C_{u p}$ is 0 . The attraction factors in other seven directions can be calculated according to the above-mentioned method. Tenlayer neighborhood is adopted to calculate the influencing factor of mainstream crowd in the paper.

(2) Mutual exclusivity of individuals

When individuals get close enough, mutual exclusivity emerges because they try to avoid contact with others. Singlelayer neighborhood is selected to analyze mutual exclusivity of individuals. Exclusivity of the cell $\mathrm{C}_{\mathrm{i}}$ 's neighbors is defined by states of their own and their neighbors. Considering the states of the eight cells in the current neighborhood, if the total number of the cells occupied by others is $\mathrm{N}_{\mathrm{r}}\left(\mathrm{N}_{\mathrm{r}}<=8\right)$, the spare cells should be $8-\mathrm{N}_{\mathrm{r}}$ and then the attraction factor impact of the cell $\mathrm{C}_{\mathrm{i}}$ with the mutual exclusivity of individuals is:

$$
F_{i}^{r}=\left(8-N_{r}\right) / 8
$$

\subsection{Rules for model evolution}

The movement of passengers is determined by the current states of the cell itself and its neighboring cells and the state of the cell is decided by the cell's attraction factor. The probability of passengers moving towards the neighboring cell $\mathrm{C}_{\mathrm{i}}$ is given by formula (8)

$$
\mathrm{PR}_{i}=\left(\alpha \bullet F_{i}^{l}+\beta \bullet F_{i}^{m}+\gamma \bullet F_{i}^{r}\right)\left(1-f_{i}\right)
$$

Where $\mathrm{PR}_{\mathrm{i}}$ is the probability for the passenger to move towards the cell $\mathrm{C}_{\mathrm{i}}$ at the next moment, and if the area where the cell $\mathrm{C}_{\mathrm{i}}$ is located is covered by walls or obstacles, or occupied by other passengers, then $f_{i}$ takes 1 ; if the cell is empty, then $f_{i}$ is set as 0 . Therein, $\alpha, \beta$ and $\gamma$ respectively indicate the weight of the three impact factor.

Transition probability can be used to determine the cell which the passenger will reach the next time. Three update modes are available: sequential update, random update, and simultaneous update. Sequential update requires that all passengers be numbered first and then states of them be updated in order. In random mode, the passenger is selected randomly for updating from those whose states are not updated yet until states of all passengers are updated. Simultaneous update used in this paper means the update of all cells' states is made at the same time. Since simultaneous update conforms to the parallel update rule, conflict occurs when several passengers compete for one position. In that case, a passenger will be randomly selected for that position from the competitors by the system and the rest will stay where they are.

\section{Simulation of the evacuation process}


According to the different purposes for simulation, the initial distribution of passengers can be divided into cyclical and boundary-fixed ones. The former is usually used to study the flow characteristics in the passageway while the latter is mostly applied to study the evacuation in a specific scenario. In this Paper, we mainly simulate the evacuation process in the passenger ship, so the boundary-fixed distribution is selected.

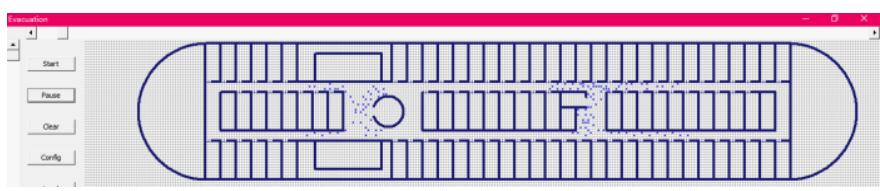

Figure 4: The simulation of evacuation process.

Figure 4 indicates the evacuation simulation achieved on the deck plane by means of $\mathrm{VC}++$, in which we can see that the whole deck is arranged in three rows and the upper and lower levels respectively are configured with 32 passenger cabins separated into two areas (front and rear) by the chimney. The middle row includes 26 passenger cabins, 2 storage rooms, 1 spiral stairs, 1 straight stair with landing and the transverse walkways.

At the start of the evacuation, assuming that the crowd is kept in passenger cabins with each one occupied by two passengers, the total number of the passengers to be evacuated should be 180. At each time step, the positions of all passengers are updated synchronously until all the passengers are evacuated from the staircase, and then the total time steps for evacuation is calculated. Figure 5 indicates the evacuation situation when the program operates at Step 53.

Table 1: The simulation result

\begin{tabular}{|c|c|c|c|}
\hline $\boldsymbol{\alpha}$ & $\boldsymbol{\beta}$ & $\boldsymbol{\gamma}$ & Time steps \\
\hline 0.5 & 0.1 & 0.4 & 202 \\
0.5 & 0.2 & 0.3 & 195 \\
0.5 & 0.25 & 0.25 & 165 \\
0.5 & 0.3 & 0.2 & 208 \\
0.5 & 0.4 & 0.1 & 237 \\
\hline
\end{tabular}

Table 1 reflects the influence of the weighting factor on the evacuation time step. When $\beta=\gamma$, the time step of the evacuation is the shortest. When $\beta<\gamma$, passengers are so concerned about the mutual exclusivity of individuals that they will find more empty way, making the evacuation time longer. When $\beta>\gamma$, passengers blindly go along with the crowd, resulting in decreased evacuation efficiency and evacuation time step became longer. Simulation results show that the mainstream crowd and the mutual exclusivity of individuals have certain influence on the evacuation process, and it is factors that must be considered in the evacuation research. When the weights are properly set, the entire evacuation process can be more realistic.

\section{Conclusion}

In this paper, the evacuation model of the passenger ship is established by the cellular automata. Making use of the advantages of the cellular automata in simulating the complicated physical phenomena, and such factors as static and dynamic floor fields are introduced. The evacuation model is rational in simulating the routes for evacuation in case of emergency, and providing support for the passenger ship design and the improvement of safety performance.

\section{References}

[1] D. Helbingp Eacute and T. Moln Aacute. "Social Force Model for Pedestrian Dynamics," Physical Review E Statistical Physics Plasmas Fluids \& Related Interdisciplinary Topics, vol. 51, pp. 4282, (1995).

[2] D. H. Rothman and S. Zaleski. "Lattice-gas models of phase separation: interfaces, phase transitions, and multiphase flow," Review of Modern Physics, vol. 66, pp. 1417-1479, (1994).

[3] V. Blue and J. Adler. "Emergent Fundamental Pedestrian Flows From Cellular Automata Microsimulation," Transportation Research Record, vol. 1644, pp. 29-36, (1998).

[4] V. J. Blue and J. L. Adler, "Cellular automata microsimulation for modeling bi-directional pedestrian walkways," Transportation Research Part B Methodological, vol. 35, pp. 293-312, (2001)

[5] M. Muramatsu, T. Irie and T. Nagatani. "Jamming transition in pedestrian counter flow," Physica A Statistical Mechanics \& Its Applications, vol. 267, pp. 487-498, (1999).

[6] K. J. Zhu and L. Z. Yang. "The effects of exit position and internal layout of classroom on evacuation efficiency," Acta Physica Sinica, vol. 59, pp. 7701-7707,(2010).

[7] Y. Hao. "Simulation of bi-directional pedestrian flow based on cellular automata model," Acta Physica Sinica Chinese Edition, vol. 57, pp. 6901-6908, (2008).

[8] S. Ha, N. K. Ku, M. I. Roh, and K. Y. Lee. "Cell-based evacuation simulation considering human behavior in a passenger ship," Ocean Engineering, vol. 53, pp. 138-152, (2012).

[9] E. Vanem and R. Skjong. "Designing for safety in passenger ships utilizing advanced evacuation analysesA risk based approach," Safety Science, vol. 44, pp. 111135, (2006).

[10] H. Kim, J. H. Park, D. Lee, and Y. S. Yang. "Establishing the methodologies for human evacuation simulation in marine accidents," Computers \& Industrial Engineering, vol. 46, pp. 725-740, (2004).

[11] K. Yoshida, M. Murayama, T. Itakaki. "Study on evaluation of escape route in passenger ships by evacuation simulation and full-scale trials," The Interflame Conf., ninth ed., Edinburgh, Scotland, (2001).

[12] W. Daamen. "Modeling passenger flows in public transport facilities, " TU Delft., Delft, pp. 37, (2004). 